\title{
Monte Carlo Simulations of Critical Dynamics with Conserved Order Parameter
}

\author{
B. Zheng \\ FB Physik, Universität Halle, 06099 Halle, Germany \\ Institute of Theoretical Physics, Academia Sinica, 100080 Beijing, P. R. China
}

\begin{abstract}
Taking the two-dimensional Ising model for example, short-time behavior of critical dynamics with a conserved order parameter is investigated by Monte Carlo simulations. Scaling behavior is observed, but the dynamic exponent $z$ is updating schemes dependent.
\end{abstract}

PACS: 64.60.Ht, 02.70.Lq, 75.10.Hk

Keywords: short-time critical dynamics, Monte Carlo simulation 
Traditionally, it is known that there exists universal dynamic scaling behavior in the long-time regime of critical dynamic evolution, since both the spatial correlation length and the correlation time are very large. In recent years, however, it is discovered that universal scaling behavior emerges already in the macroscopic short-time regime, after a microscopic time scale $t_{m i c}$ which is large enough in the microscopic sense [1, 2, 3, 4, 5, 6, 7]. Important is that one should take care of the macroscopic initial conditions carefully.

For a random initial state, for example, at the critical point the nonequilibrium spatial correlation length increases in time by a power law $t^{1 / z}$ due to the divergent correlation time [7]. This implies a kind of memory effect in the system. Even though the spatial correlation length $\left(\sim t^{1 / z}\right)$ is still small in the short-time regime, one may expect dynamic scaling. For a small initial magnetization, an extra critical exponent is sufficient to describe its scaling behavior [1, 5, []. In general, one needs a characteristic function for an arbitrary magnetization [8, 9]. If the initial spatial correlation length is non-zero, it induces corrections to scaling [1].

Since 1989, analytical calculations for short-time dynamic scaling behavior based on renormalization group methods [1, 10, 11] have extended to different dynamics classified by Hohenberg and Halperin [12]. However, Monte Carlo simulations are still limited in dynamics of model A [0, 13, 14, 15, 16, 17, 18, 19, 20, 21]. Actually, due to severe critical slowing down, Monte Carlo simulations for long-time behavior of critical dynamics beyond model A are also in preliminary stage. On the other hand, short-time dynamic scaling is not only conceptually interesting but also practically important. It provides new methods for numerical measurements of not only dynamic exponents but also static exponents as well as the critical temperature [22, 23]. These methods do not suffer from critical slowing down. Therefore it is important to investigate short-time scaling behavior of critical dynamics beyond model A with Monte Carlo methods.

Dynamics of model A is a kind of relaxation dynamics without relevant conserved quantities [12]. If we consider only dynamic relaxation processes, dynamics with different relevant conserved quantities is classified into model $\mathrm{B}, \mathrm{C}$ and $\mathrm{D}$. In this letter, taking the two-dimensional Ising model for example, with Monte Carlo methods we study dynamics with a conserved order parameter, which is called model B, according to Ref. [12].

In dynamics of model $\mathrm{B}$, to guarantee criticality the conserved order pa- 
rameter must be set to zero. The equilibrium state of model $\mathrm{B}$ is in a same universality class of model A. For lattice models, the critical temperature in dynamics of model $\mathrm{B}$ is also the same as that of model A. Of course, the dynamic exponent $z$ of model B is different from that of model A. For the $\phi^{4}$ theory with a generalized Langevin equation in which the order parameter is conserved, in the long-time regime of dynamic evolution it has been derived that $z=4-\eta$, with $\eta$ being the well known static exponent [12]. In Ref. [回], it is argued that there exists also short-time dynamic scaling and no new independent critical exponents emerge.

For the Ising model, a realization of dynamics of model $\mathrm{B}$ is considered to be Kawasaki dynamics in which two neighboring spins exchange their values in a flip. Early simulations with small lattices for dynamic behavior in equilibrium yield a dynamic exponent $z$ consistent with the theoretical prediction $z=4-\eta$ [24]. In this paper, we study the non-equilibrium dynamic process starting from a random initial state. We measure the spatial correlation function

$$
C(r, t)=\frac{1}{L^{d}} \sum_{i}\left\langle S_{i}(t) S_{i+r}(t)\right\rangle
$$

Here $S_{i}$ is a Ising spin, $d=2$ is the space dimension and $L$ is the lattice size. In updating, we randomly pick up neighboring spin pairs. When $L^{d}$ pairs of spins are updated, we define one Monte Carlo time step. At the critical point, standard scaling form for $C(r, t)$ is written as

$$
C(r, t)=t^{-2 \beta / \nu z} F\left(\frac{r}{t^{1 / z}}\right) .
$$

Here $\beta$ and $\nu$ are static exponents and $z$ is the dynamic exponent. Usually such a scaling form is assumed to be valid already in the macroscopic shorttime regime, after a time scale $t_{m i c}$ which is large enough in the microscopic sense. If a Monte Carlo time step is considered as a typical microscopic time unit, $t_{\text {mic }}$ may be several to some hundred time steps. For dynamics of model A, this is indeed the case [7].

In Fig. 1, the spatial correlation function $C(r, t)$ for Kawasaki dynamics with the heat-bath algorithm is displayed. The lattice size is $L=512$ and samples of initial configurations for averaging are 100. Maximum updating time is 64000. Here finite size effect is apparently negligible. For example, 
$C(r, t)$ at $r=80$ is already very small. Extra simulations up to $t=256000$ show similar behavior as in Fig. 1, but one needs bigger lattices.

Carefully looking at Fig. 1, we conclude that there is no scaling behavior as described by Eq. (2), even though it seems there is a kind of self-similarity during the dynamic evolution. Let us concentrate our attention on the first peak in $r$ direction (for a fixed time $t$ ). If there would be scaling, the first peak for different time $t$ 's should overlap each other after properly rescaling $r$ and $C(r, t)$ according to Eq. (2). We remind ourselves that the exponent $2 \beta / \nu z$ is positive. Therefore the peak for a larger $t$ should be lower than that for a smaller $t$. But it is not the case in Fig. 1. This fact indicates that at least for the short-time behavior, Kawasaki dynamics of the Ising model can not be described by the generalized Langevin equation of model B for the $\phi^{4}$ theory given in Ref. [12].

Why there exists no short-time dynamic scaling behavior in Kawasaki dynamics is not very clear for us. What we observe is that in Kawasaki dynamics the order parameter is conserved very locally by exchanging two neighboring spins. Spin exchanging takes place only along domain walls. Smaller domains can not be so easily created during time evolution. As a result, the distribution of domain sizes (at a fixed time $t$ ) is rather sharp. This is the origin of the oscillating mode in $C(r, t)$ as shown in Fig. 1. This oscillating mode violates the scaling form.

Dynamics with a conserved order parameter is not unique. For example, we may consider an alternative dynamic process in which the order parameter is only globally conserved. A simple case is that we exchange two randomly separated spins. In this case, scaling behavior is observed. To show the scaling behavior is rather robust, we have performed the simulations with different updating schemes and algorithms. In Fig. 2 (a), correlation functions $C(r, t)$ with the heat-bath algorithm are displayed for two different updating schemes. Solid lines are from a scheme in which one spin sweeps over the lattice regularly, while dashed lines are from a scheme in which both spins are randomly selected. Total samples of initial configurations for averaging are 620 and 220 respectively. Obviously, both updating schemes yield almost the same results, even though some fluctuation is seen for larger times. In Fig. 2 (b), similar curves are seen for both the heat-bath and the Metropolis algorithms. But Metropolis dynamics evolves slightly faster. Important in Fig. 2 is that the oscillating mode is swept away. Comparing Fig. 11 and 2 , we observe that Kawasaki dynamics is much slower. 
Now we study scaling behavior of $C(r, t)$. According to Eq. (2), if the dynamic exponent $z$ and the static exponent $\beta / \nu$ are properly chosen, $C(r, t)$ at different time $t$ 's collapse on a single curve. In other words, from scaling collapse of $C(r, t)$ one can estimate $z$ and $\beta / \nu$. Very careful analysis shows that the microscopic time scale $t_{m i c}$ 's for the heat -bath and the Metropolis algorithms are less than 320 and 160 respectively. Also, up to the time $t=5120$ there exists no visible finite size effect for both algorithms with $L=512$. In Fig. 3, scaling plot for $C(r, t)$ is shown. All data points collapse nicely. The fitted exponents are $z=2.315(5)$ and $2 \beta / \nu=0.252(3)$ for the heat-bath algorithm and $z=2.330(15)$ and $2 \beta / \nu=0.251(2)$ for the Metropolis algorithm. Results for both algorithms are well consistent. The values for the exponent $2 \beta / \nu$ agree remarkably with the exact value 0.25 obtained in equilibrium. These results strongly support the scaling form in Eq. (2).

If we fix the static exponent $2 \beta / \nu$ to its exact value in the scaling collapse, the resulting dynamic exponent $z$ is $z=2.320(5)$ for the heat-bath algorithm and $z=2.335(10)$ for the Metropolis algorithm. Taking into account all above measurements, we conclude

$$
z=2.325(10)
$$

This value of $z$ is significantly different from $z=4-\eta=3.75$. In other words, the dynamics is not in a same universality class of the $\phi^{4}$ theory with the generalized Langevin equation in Ref. [12]. The value $z=2.325(10)$ is relatively close to $z=2.165$ (10) for dynamics of model A [7], but also clearly different.

Another interesting dynamic observable is the auto-correlation function

$$
A(t)=\frac{1}{L^{d}} \sum_{i}\left\langle S_{i}(0) S_{i}(t)\right\rangle
$$

In Refs. [25, 1], it has been derived that at the critical point, for dynamics of model A, $A(t)$ decays by a power law

$$
A(t) \sim t^{-\lambda}
$$

Here the exponent $\lambda=d / z-\left(2-z-\eta-\eta_{0} / 2\right) / z$, and $\eta_{0}$ is an exponent induced from the divergence related to the initial condition [25, 1]. For dynamics of 
model B for the $\phi^{4}$ theory discussed in Ref. [12], it is pointed out in Ref. [1], $\eta_{0}=0$, since there is no divergence related to the initial condition.

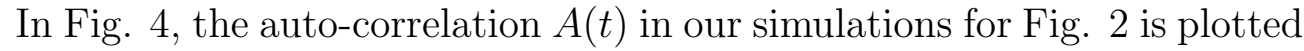
in double-log scale. Power law behavior is observed starting around a time $t=20$. After $t=1000$, fluctuation becomes large. In the time interval $[20,1000]$, we measure the exponent $\lambda=0.675(3)$ and $0.679(13)$ for the heatbath and the Metropolis algorithms respectively. As an average, we estimate

$$
\lambda=0.677(8) \text {. }
$$

Obviously, it does not yield $\eta_{0}=0$ and also is different from $\lambda=0.737(1)$ for dynamics of model A [7].

In conclusions, we have investigated short-time behavior of critical dynamics for the two-dimensional Ising model with a conserved order parameter. For a locally conserved order parameter (Kawasaki dynamics), standard scaling behavior does not exist. If we release the condition such that the order parameter is only globally conserved, universal scaling behavior emerges. However, the dynamics is not in a same universality class of the $\phi^{4}$ theory with the generalized Langevin equation which is called model B in Ref. [12]. Very probably both $z$ and $\lambda$ are independent critical exponents in this case. It is actually interesting how to construct a corresponding continuum model and solve it with renormalization group methods. It seems that there might be some similarity at certain points between this dynamics and kinetic roughening [26].

\section{Erratum}

We regret that the data for Fig. 1 are obtained with an incorrect updating scheme. The discussions and conclusions based on Fig. 1 must be modified. A correct version of Fig. 1 is displayed in Fig. 5. The scaling behavior described by Eq. (1) is observed. The dynamic exponent $z$ depends slightly on the time $t$. From our data, we could only conclude that $z$ is 3.95(10). This is somewhat bigger than the theoretical value $z=4-\eta=3.75$ calculated with the $\phi^{4}$ theory. Probably there exist still corrections to scaling. To perform simulations to larger time $t$ 's and remove these corrections to scaling, 
however, is not so easy since $z$ has a big value. From the auto-correlation, we

estimate the exponent $\lambda=0.495(10)$. It is consistent with the scaling relation $\lambda=d / z$ derived with the $\phi^{4}$ theory (formally $\lambda=d / z-\left(4-z-\eta-\eta_{0} / 2\right) / z=$ $d / z+\eta_{0} / 2 z$, but $\left.\eta_{0}=0\right)$.

Acknowledgment: The author thanks for the visiting scholarship and the hospitality from Institute of Theoretical Physics. This work is supported in part by DFG, Az. TR 300/3-1.

\section{References}

[1] H. K. Janssen, B. Schaub and B. Schmittmann, Z. Phys. B 73 (1989) 539.

[2] D. A. Huse, Phys. Rev. B 40 (1989) 304.

[3] D. Stauffer, Physica A 186 (1992) 197.

[4] Z.B. Li, U. Ritschel and B. Zheng, J. Phys. A27 (1994) L837.

[5] L. Schülke and B. Zheng, Phys. Lett. A 204 (1995) 295.

[6] P. Grassberger, Physica A 214 (1995) 547.

[7] B. Zheng, Int. J. Mod. Phys. B12 (1998) 1419, review article.

[8] B. Zheng, Phys. Rev. Lett. 77 (1996) 679.

[9] Y. Chen, S. H. Guo, Z. B. Li and A. Ye, Euro. Phys. J. B B15 (2000) 97.

[10] K. Oerding and H. K. Janssen, J. Phys. A26 (1993) 3369,5295.

[11] K. Oerding and H. K. Janssen, J. Phys. A27 (1994) 715.

[12] P.C. Hohenberg and B.I. Halperin, Rev. Mod. Phys. 49 (1977) 435.

[13] J. F. F. Mendes and M. A. Santos, Phys. Rev. E57 (1998) 108.

[14] T. Tomé and M. J. de Oliveira, Phys. Rev. E58 (1998) 4242.

[15] A. Brunstein and T. Tomé, Phys. Rev. E60 (1999) 3666.

[16] B. Zheng, M. Schulz and S. Trimper, Phys. Rev. E59 (1999) R1351.

[17] H.J. Luo, L. Schülke and B. Zheng, Mod. Phys. Lett. B13 (1999) 417.

[18] J. B. Zhang, L. Wang, D. W. Gu, H. P. Ying and D. R. Ji, Phys. Lett. A262 (1999) 226. 
[19] A. J. Bray, A. J. Briant and D. K. Jervis, Phys. Rev. Lett. 84 (2000) 1503.

[20] L. M. Jensen, B. J. Kim and P. Minnhagen, Phys. Rev. B61 (2000) 15412.

[21] H. P. Ying and K. Harada, Phys. Rev. E62 (2000) 174.

[22] Z.B. Li, L. Schülke and B. Zheng, Phys. Rev. Lett. 74 (1995) 3396.

[23] H.J. Luo, L. Schülke and B. Zheng, Phys. Rev. Lett. 81 (1998) 180.

[24] M. C. Yalabik and J. D. Gunton, Phys. Rev. B25 (1982) 534.

[25] H. K. Janssen, in From Phase Transition to Chaos, edited by G. Györgyi, I. Kondor, L. Sasvári and T. Tél, Topics in Modern Statistical Physics (World Scientific, Singapore, 1992).

[26] J. M. López, Phys. Rev. Lett. 83 (1999) 4594. 


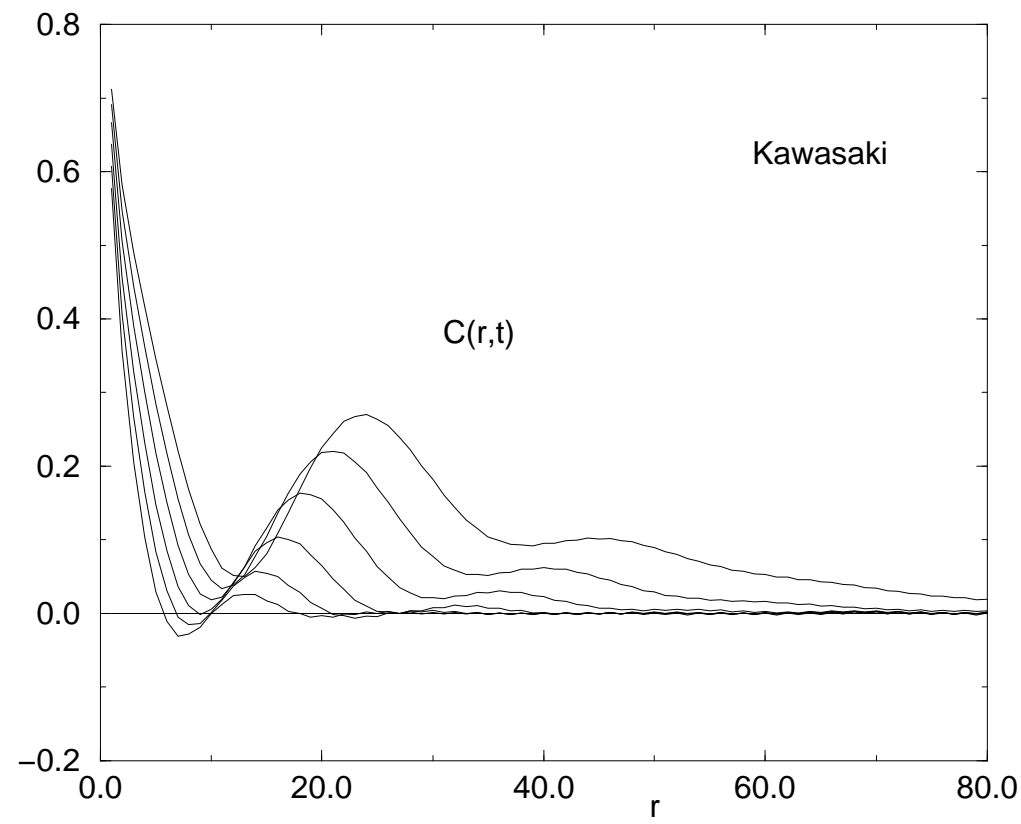

Figure 1: Correlation functions $C(r, t)$ for Kawasaki dynamics with the heatbath algorithm. The lattice size is $L=512$. From left to right, curves correspond to the time $t=2000,4000,8000,16000,32000$ and 64000 . 

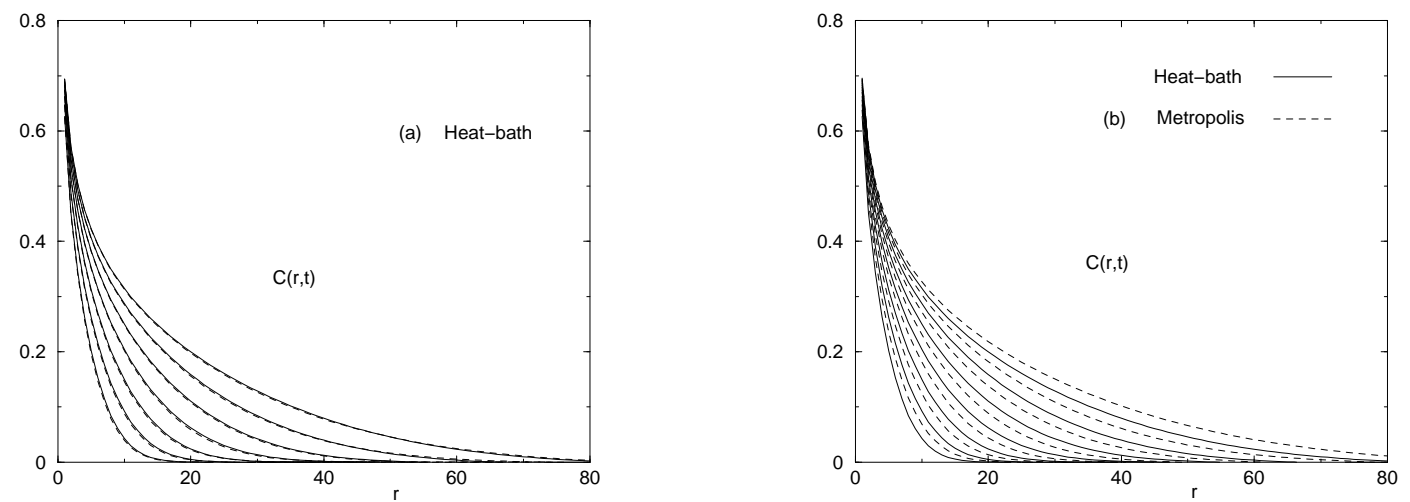

Figure 2: Correlation functions $C(r, t)$ for dynamics with a globally conserved order parameter. The lattice size is $L=512$. (a) The heat-bath algorithm. Solid lines are from a updating scheme in which one spin sweeps over the lattice regularly. Dashed lines are from a scheme in which both spins are randomly selected. (b) The solid lines are the same as in (a) but dashed lines are obtained with the Metropolis algorithm. In both (a) and (b), from left to right, curves correspond to the time $t=80,160,320,640,1280,2560$ and 5120 . 

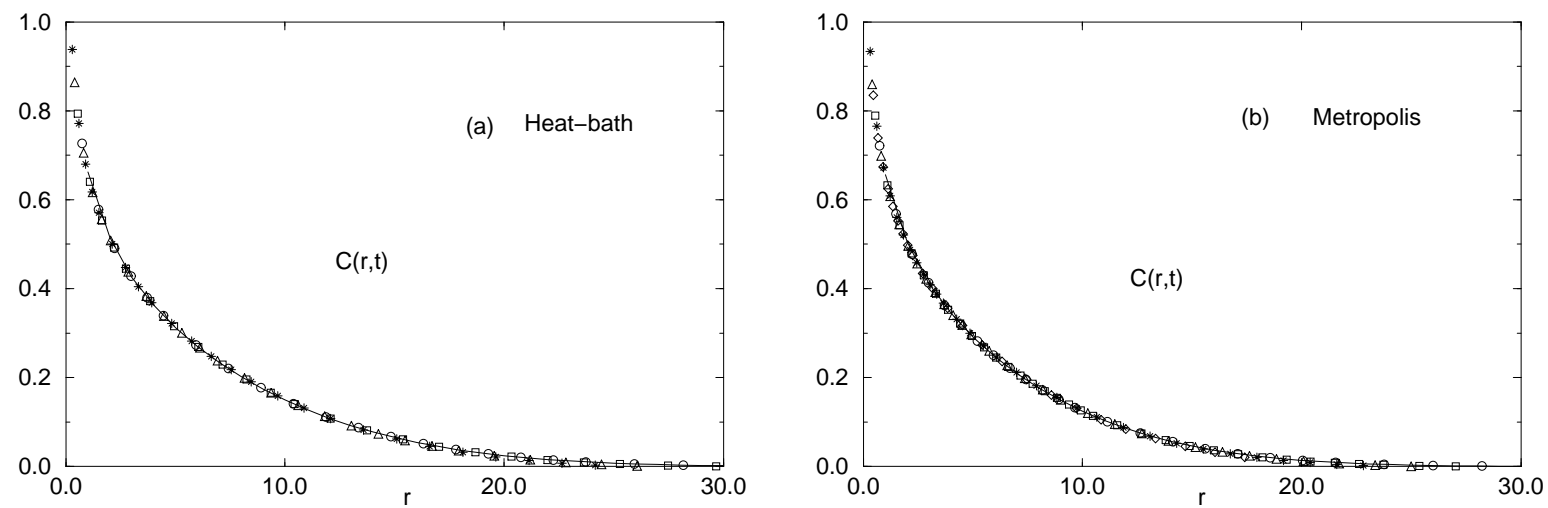

Figure 3: Scaling plot for $C(r, t)$. (a) The solid line is for $t_{1}=320$, while $\circ, \square, \triangle$ and $*$ fitted to the curve of $t_{1}=320$ are those for $t=640,1280$, 2560 and 5120 but $r$ and $C$ are rescaled according to Eq. (2) to $r /\left(t / t_{1}\right)^{1 / z}$ and $\left(t / t_{1}\right)^{2 \beta / \nu z} C$ with $z=2.315$ and $2 \beta / \nu=0.252$. (b) The solid line is for $t_{1}=160$, while $\circ, \square, \triangle, *$ and $\diamond$ are for $t=320, t=640,1280,2560$ and 5120 with $z=2.330$ and $2 \beta / \nu=0.251$. 


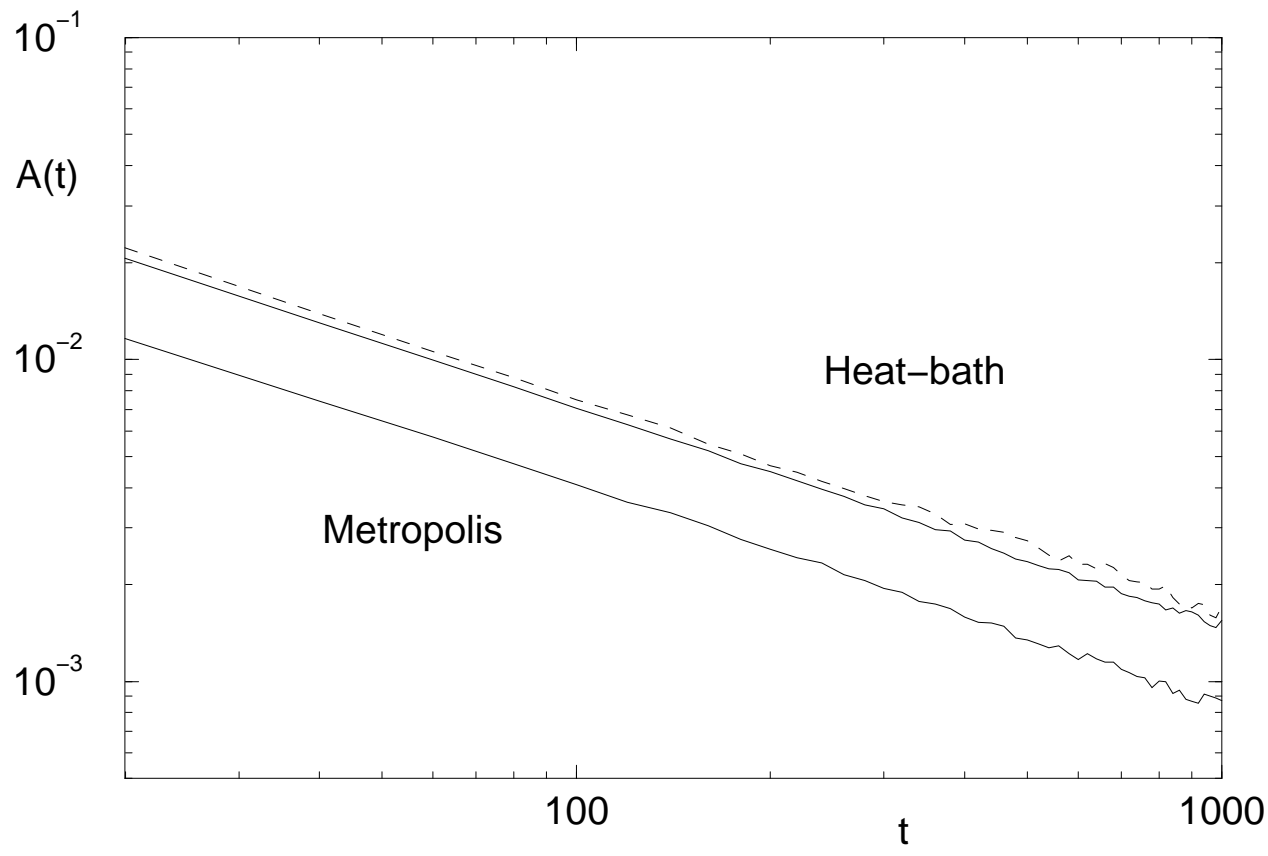

Figure 4: Auto-correlation $A(t)$ in double-log scale. The dashed line is for the updating scheme with both spins randomly picked up 


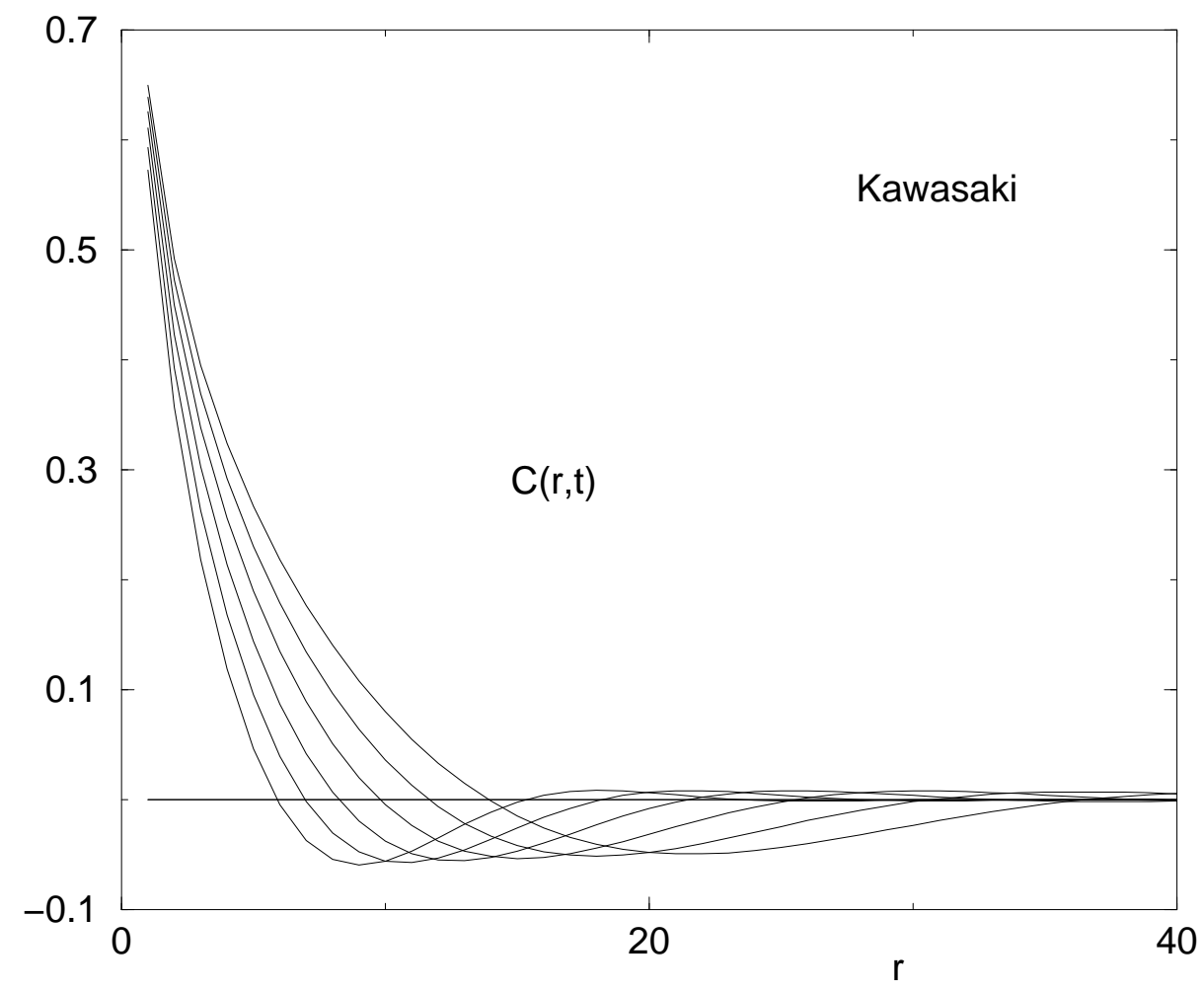

Figure 5: Correlation functions $C(r, t)$ for Kawasaki dynamics with the heatbath algorithm. The lattice size is $L=512$. From left to right, curves correspond to the time $t=2000,4000,8000,16000,32000$ and 64000 . 